\section{BRAZIULIAN JOURNAL}

OF MEDICAL AND BIOLOGICAL RESHARCH

www.bjournal.com.br
ISSN 0100-879X

Volume 43 (01) 1-123 January 2010

BIOMEDICAL SCIENCES

AND

CLINICAL INVESTIGATION

Braz J Med Biol Res, J anuary 2010, Volume 43(1) 1-7

Genetics of homocysteine metabolism and associated disorders

S. Brustolin, R. Giugliani and T.M. Félix

The Brazilian Journal of Medical and Biological Research is partially financed by
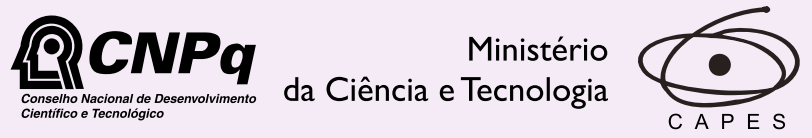

Ministério da Educação

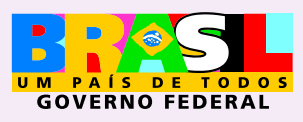

Institutional Sponsors 


\title{
Genetics of homocysteine metabolism and associated disorders
}

\author{
S. Brustolin ${ }^{1}$, R. Giugliani ${ }^{1,2}$ and T.M. Félix ${ }^{1}$
}

${ }^{1}$ Serviço de Genética Médica, Hospital de Clínicas de Porto Alegre, Porto Alegre, RS, Brasil

2Departamento de Genética, Universidade Federal do Rio Grande do Sul, Porto Alegre, RS, Brasil

\begin{abstract}
Homocysteine is a sulfur-containing amino acid derived from the metabolism of methionine, an essential amino acid, and is metabolized by one of two pathways: remethylation or transsulfuration. Abnormalities of these pathways lead to hyperhomocysteinemia. Hyperhomocysteinemia is observed in approximately $5 \%$ of the general population and is associated with an increased risk for many disorders, including vascular and neurodegenerative diseases, autoimmune disorders, birth defects, diabetes, renal disease, osteoporosis, neuropsychiatric disorders, and cancer. We review here the correlation between homocysteine metabolism and the disorders described above with genetic variants on genes coding for enzymes of homocysteine metabolism relevant to clinical practice, especially common variants of the MTHFR gene, 677C $>\mathrm{T}$ and $1298 \mathrm{~A}>\mathrm{C}$. We also discuss the management of hyperhomocysteinemia with folic acid supplementation and fortification of folic acid and the impact of a decrease in the prevalence of congenital anomalies and a decline in the incidence of stroke mortality.
\end{abstract}

Key words: Homocysteine; Hyperhomocysteinemia; Folate metabolism; Single nucleotide polymorphism; Susceptibility genes

\section{Introduction}

Homocysteine (Hcy) is the demethylated derivative of methionine, which, after conversion to S-adenosylmethionine, is the most important methyl group donor in the body. Hcy is metabolized by one of the two following pathways, i.e., remethylation or transsulfuration (Figure 1). In remethylation, Hcy is transformed to methionine by the addition of a methyl group from 5-methyltetrahydrofolate or betaine. 5-Methyltetrahydrofolate is the result of the conversion of folic acid from the diet to 5,10-methyltetrahydrofolate and finally to 5-methyltetrahydrofolate by the enzyme 5,10-methyltetrahydrofolate reductase (MTHFR). In all tissues, the cofactor vitamin B12 participates in the remethylation reaction with 5-methyltetrahydrofolate, whereas the reaction with betaine is restricted to the liver and is independent of vitamin B12. Methionine is then activated by ATP to form Sadenosylmethionine, a universal methyl donor to a variety of acceptors. In the transsulfuration pathway, Hcy is converted to cystathionine by cystathionine $\beta$-synthase and finally to cysteine using vitamin B6 as a cofactor (1).

Therefore, plasma Hcy levels are determined by several factors such as the cofactors vitamin B12, vitamin B6 and folic acid and enzymes involved in methionine metabolism. The levels of these cofactors are inversely correlated, with plasma Hcy levels increasing with decreasing vitamin B, folic acid and vitamin B6 concentrations (1).

In the circulation, $80-90 \%$ of Hcy is protein bound especially to albumin, its main carrier in plasma. The remaining $10-20 \%$ of total Hcy (tHcy) is acid-soluble free Hcy presenting as Hcy-cysteine mixed disulfide and homocystine (a dimer of Hcy) and less than the remaining $1 \%$ is present in the free reduced form (2).

\section{Homocysteine and MTHFR}

MTHFR is an important enzyme in Hcy metabolism, which catalyzes the conversion of 5,10-methylenetetrahydrofolate to 5-methyltetrahydrofolate, the predominant circulating form of folate. The MTHFR gene has at least two functional polymorphisms, $677 \mathrm{C}>\mathrm{T}$ and $1298 \mathrm{~A}>\mathrm{C}$. The MTHFR 677T allele is associated with reduced enzymatic activity, decreased concentrations of folate in serum, plasma, and red blood cells, and mildly increased plasma tHcy concentrations $(3,4)$.

The MTHFR polymorphism 1298A $>\mathrm{C}$ also affects MTHFR activity but is not associated with higher plasma Hcy or lower folate levels (5). Normal MTHFR activity is

Correspondence: T.M. Félix, Serviço de Genética Médica, Hospital de Clínicas de Porto Alegre, Rua Ramiro Barcelos, 2350, 90035903 Porto Alegre, RS, Brasil. Fax: +55-51-3359-8010. E-mail: tfelix@hcpa.ufrgs.br

Received April 29, 2009. Accepted November 26, 2009. Available online December 7, 2009. Published January 11, 2010. 


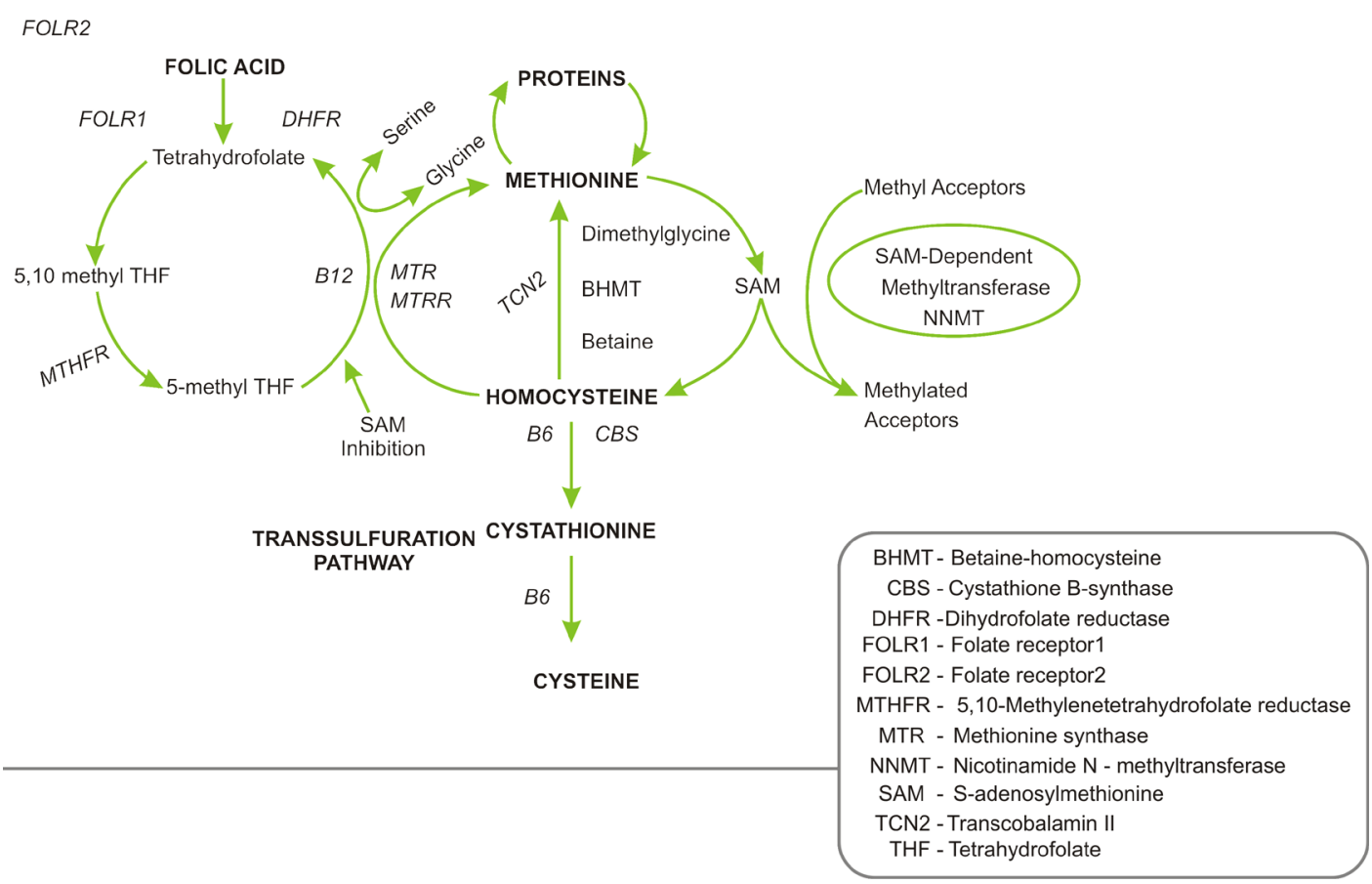

Figure 1. Homocysteine metabolism.

crucial to maintain the pool of circulating folate and methionine and to prevent the accumulation of Hcy (3).

Double heterozygozity for MTHFR $677 \mathrm{C}>\mathrm{T}$ and 1298A > C polymorphisms results in lower MTHFR activity as compared to heterozygosity for either MTHFR variant separately (5). Individuals with the 677TT genotype have approximately $30 \%$ the MTHFR enzyme activity of those with the $677 \mathrm{CC}$ genotype, whereas $677 \mathrm{CT}$ heterozygotes have about $65 \%$ enzymatic activity (6).

\section{Hyperhomocysteinemia}

Blood levels of hyperhomocysteinemia (HHcy) are age and gender related. Plasma Hcy levels are higher in men than in women (7) and increase from $10.8 \mu \mathrm{mol} / \mathrm{L}$ at age $40-42$ up to $12.4 \mu \mathrm{mol} / \mathrm{L}$ between $65-67$ years (8). HHcy is classified as moderate (15-30 $\mu \mathrm{mol} / \mathrm{L})$, intermediate (31-100 $\mu \mathrm{mol} / \mathrm{L})$ or severe $(>100 \mu \mathrm{mol} / \mathrm{L})(9)$.

In humans, HHcy can also be distinguished by category, including cause, prevalence and severity. The more severe cases are due to homozygous defects in genes encoding enzymes of Hcy metabolism (Table 1).

$\mathrm{HHcy}$ is observed in approximately $5 \%$ of the general population and has been associated with many disorders. Below we review several disorders that have been related to abnormal Hcy metabolism.

Table 1. Classification of hyperhomocysteinemia according to Selhub (1).

\footnotetext{
Severe hyperhomocysteinemia

High total homocysteine (tHcy) levels at all times (31 to $>100 \mu \mathrm{mol} / \mathrm{L}$ ) caused, for example, by deficiencies in cystathionine beta synthase (CBS), methylenetetrahydrofolate reductase (MTHFR), or in enzymes of B12 metabolism

Mild hyperhomocysteinemia

Moderately high tHcy levels (15-30 $\mu \mathrm{mol} / \mathrm{L})$ under fasting conditions; reflects impaired homocysteine methylation (folate, B12 or moderate enzyme defects, e.g., thermolabile MTHFR)

Post-methionine load

Abnormal increase in tHcy (>15 $\mu \mathrm{mol} / \mathrm{L})$ after a methionine load (100 mg/kg); reflects impaired homocysteine transsulfuration (heterozygous CBS defects, B6 deficiency)
} 


\section{Hyperhomocysteinemia and neural tube defects}

Neural tube defects (NTD) are among the most common birth defects worldwide. NTD result from failure of neural tube formation or closure during the first 28 days of pregnancy. Studies have supported the use of folic acid in the prevention of the first occurrence of NTD (10). These findings suggest that impaired folic acid metabolism plays a role in NTD.

Several studies on different populations have reported that high maternal Hcy levels are associated with an increased risk for offspring with NTD (11-17). A case-control study conducted in Brazil showed that mothers with NTD children had increased tHcy levels when compared with control mothers. Case mothers also had low vitamin B12 levels compared to control mothers. No differences in MTHFR polymorphisms were identified in mothers, children with NTD or controls (18). Another Brazilian study was also unable to identified MTHFR polymorphism and NTD, suggesting that this gene does not play a role in NTD in the Brazilian population (19).

\section{Hyperhomocysteinemia and nonsyndromic oral clefts}

Oral clefts are common birth defects whose prevalence differs between geographical regions and ethnic groups. They have a complex, multifactorial inheritance involving genetic and environmental factors.

A case-control study showed that mothers of children with clefts had higher plasma tHcy concentrations both under fasting conditions and after a methionine afterload test compared to control mothers, suggesting that impaired folic acid metabolism plays a role in clefting (20). A recent study has indicated that periconceptional use of folate could prevent the occurrence of cleft lip and palate (CL/P) but not of cleft palate alone (21).

A study demonstrated a significantly increased risk of having a child with CL/P if the mother was heterozygous for both common MTHFR polymorphisms (677C>T, 1298A>C) (22). The risk of a child with CL/P was only significantly increased if mothers carrying the MTHFR 677TT or MTHFR $1298 C C$ genotype also had a low periconceptional intake of dietary folate and/or folic acid supplements, suggesting that the two MTHFR polymorphisms were independent risk factor for CL/P (23).

\section{Hyperhomocysteinemia and congenital heart defects}

Congenital heart defects (CHD) occur in 11/1000 live births in the United States and annually account for approximately 6000 total deaths and one-tenth of infant deaths in the USA. CHD develop during the first 3 weeks after conception and are common congenital anomalies of multifactorial origin influenced by genetic and environmental factors (24). A meta-analysis demonstrated that maternal $\mathrm{HHcy}$ was significantly associated with an increased risk of having a child with a CHD, suggesting that maternal HHcy is an important risk factor for CHD (23).

\section{Hyperhomocysteinemia and cardiovascular disorders}

Vascular diseases are commonly associated with traditional risk factors such as systemic arterial hypertension, diabetes mellitus and smoking, but in the last decade other risk markers have been identified, one of them being Hcy. Various studies had identified HHcy as an independent risk factor for coronary artery disease (CAD). The relationship between MTHFR polymorphism and the severity of CAD in patients undergoing coronary artery bypass surgery showed that Hcy levels were significantly higher in patients with CAD than in control subjects and the genotype of MTHFR $677 \mathrm{C}>\mathrm{T}$ was associated with the extent of CAD in patients at high risk for this pathology (25).

A study comparing post-mortem serum Hcy levels of men who died suddenly of severe CAD and controls showed that serum Hcy levels were elevated in men who died with coronary thrombus compared to controls. This study also showed that the risk was increased if there was concomitant diabetes mellitus and the presence of fibrous plaque atheromas. Whether elevated serum Hcy might be causally associated with sudden death or merely a marker of a process related to sudden death is still unclear (26).

\section{Hyperhomocysteinemia and atherosclerosis}

Atherosclerosis is a chronic inflammatory disease of the arteries, in which deposits of fatty substances, cholesterol, calcium and other substances build up in the endothelial layer of the arteries. Several studies have identified moderate $\mathrm{HHcy}$ as an independent risk factor for atherosclerotic disease (9).

Accelerated atherosclerosis had been demonstrated in apolipoprotein E-deficient (apoE ${ }^{-/-}$) mice on an Hcy diet and in cystathionine-synthase (CBS)/apoE-/- double-knockout mice $(27,28)$. These findings suggest that HHcy accelerates atherogenesis in a hyperlipidemic mouse model that develops spontaneous atherosclerosis. However, a causal relationship between atherogenesis and $\mathrm{HHcy}$ had not been established in other species, including rats, rabbits, pigs, and primates $(29,30)$.

\section{Hyperhomocysteinemia and Down syndrome}

Down syndrome (DS) occurs due to three copies of 
chromosome 21 , in most cases due to the failure of chromosomal segregation during maternal meiosis. The risk of DS is directly correlated to increased maternal age. An important factor that could modulate the maternal risk for DS is plasma HHcy concentration. An elevated risk for DS has been observed in the presence of the MTR 2756G allele in combination with elevated HHcy concentration (31). However, the association of this polymorphism with the risk for DS was not confirmed by an additional study (32).

Astudy conducted in Brazil with mothers of children with DS and control mothers analyzed tHcy and several variants in the folate pathway (MTHFR 677C > T, MTHFR 1298A >C, MTRR 66A>G, MTR 2756A>G, and CBS 844ins68), showing that Hcy levels were higher among DS mothers than among controls. Only the 677T allele was associated with altered tHcy levels in the case group. All genotype distributions were similar in the two groups, but the frequency of the $677 \mathrm{~T}$ allele was significantly higher in the case group. None of the other polymorphisms showed an association with risk for DS when evaluated separately. However, when the presence of the alleles was evaluated as a whole, the mothers of children with DS tended to have a higher number of variant alleles than control mothers (33).

When the effect of plasma HHcy concentrations on maternal risk for DS was investigated, Hcy concentrations were found to be significantly higher in DS mothers compared to control. The study also showed that Hcy concentrations were significantly higher in DS mothers with the MTHFR 1298CC genotype compared to the same genotype of control mothers (34).

\section{Hyperhomocysteinemia and smoking or pregnancy}

Cigarette smoking has been associated with oxidative stress and an increased risk of many chronic diseases. Smoking induces depletion of cellular antioxidants and is also known to be associated with increased Hcy levels. Several studies have observed an increase in plasma tHcy among smokers compared to non-smokers $(35,36)$.

During pregnancy there is an increase in Hcy levels for each trimester of pregnancy and a decrease of folate levels. Additionally, folate levels continue to decrease after pregnancy (37). Maternal smoking also alters total Hcy concentration in infants. Serum Hcy concentrations were significantly higher in smoking than non-smoking pregnant women and also in the umbilical cord blood of their newborns (38).

\section{Hyperhomocysteinemia and Alzheimer's disease and vascular dementia}

Low folate and increased Hcy concentrations in blood are associated with poor cognitive performance in the general population. Elevated levels of Hcy result in neurotoxic and vasotoxic effects in dementia and Alzheimer's disease, suggesting that Hcy is a direct marker of early cognitive decline (39). However, the pathogenesis of HHcy is still not clear in vascular dementia. The levels of serum folate, vitamin B12 and plasma Hcy were studied in vascular dementia and Alzheimer's disease, and it was found that Hcy was increased, while folate and vitamin B12 were decreased in both of these diseases. This suggests that supplementation of folic acid and vitamin B12 could be of benefit in Alzheimer's disease and dementia (40).

A randomized, double-blind, placebo-controlled study was conducted in the Netherlands on 818 subjects, men and post-menopausal women aged $50-70$ years who used placebo or $800 \mu \mathrm{g} /$ day folic acid for 3 years. Serum folate concentrations increased and plasma Hcy concentrations decreased in participants taking folic acid compared with those taking placebo. This study also showed that supplementation with folic acid significantly improved the decline of cognitive function related to age (41).

\section{Hyperhomocysteinemia and breast cancer}

A high intake of folate, which is plentiful in vegetables and fruits, has been associated with reduced risk of several cancers. Folate deficiency has been suggested to increase the risk of cancer through impaired DNA repair synthesis and disruption of DNA methylation that may lead to protooncogene activation (42). It is biologically plausible that polymorphisms or gene-environment interactions rather than folate intake alone would have an impact on breast cancer risk since functional polymorphisms in folate-related genes contribute to the alteration of folate metabolism. Therefore, MTHFR polymorphisms have been intensively studied in breast cancer but the results are inconsistent $(43,44)$.

However, in a study performed with 456 breast cancer cases and 912 controls, breast cancer risk was inversely associated with consumption of dietary folate and none of the polymorphisms (MTHFR, MTRR, MTR) showed any significant impact on breast cancer risk. In postmenopausal women, a significantly increased risk of breast cancer was found among individuals with the MTHFR 677TT genotype compared with those with the MTHFR 677CC genotype. The study showed a significant interaction between MTRR A66G and folate intake. The MTRR 66GG genotype increased the risk among postmenopausal women with a low folate intake (45).

\section{Hyperhomocysteinemia and depression}

Folate and vitamin B12 deficiency, HHcy and the 677T allele of the MTHFR gene, which cause impaired methylation reactions in the central nervous system, have been associated with depressive disorders (46). However other studies did not find this association $(47,48)$. 
A prospective study on 732 Korean subjects investigated associations between folate, vitamin B12, Hcy, and latelife depression. The incident depression was predicted by lower folate and vitamin B12 levels and higher Hcy levels determined 2 years previously, and was associated with a decline in vitamin B12 levels and an increased in Hcy levels over the intervening period. However, incident depression was not associated with MTHFR genotype (49).

\section{Hyperhomocysteinemia and diabetes}

Mild HHcy has been observed in type I diabetic patients with microalbuminuria and nephropathy and may explain the increased risk of vascular disease in this high-risk population (50).

Astudy conducted in Spain on 46 patients with diabetes mellitus between 4 and 19 years of age showed that children with strict control of diabetes type I had normal tHcy, with no significant difference compared to healthy individuals of the same age and social environment (51). It was hypothesized that oral administration of folic acid would reduce plasma Hcy levels, improving endothelial function and oxidative stress in patients with type I diabetes and microalbuminuria. A case study showed a $25 \%$ reduction in plasma Hcy after folic acid supplementation in diabetic subjects (52).

\section{Hyperhomocysteinemia and drugs}

There is evidence supporting the unfavorable effects of some anti-epileptic drugs on plasma Hcy concentrations. Approximately $10-40 \%$ of epileptic patients develop HHcy (53). The levels of HHcy were increased in monotherapy consisting of phenytoin, carbamazepine or valproic acid (54).

A prospective observational study was conducted in Italy in 2007 to evaluate the influence of antiepileptic drugs, MTHFR polymorphism and Hcy levels. A higher prevalence of the MTHFR $1298 \mathrm{C}$ allele was observed in epileptic patients compared to controls. After folate therapy, plasma tHcy and folate were normal in all patients (55).

\section{Management of hyperhomocysteinemia}

Elevations in plasma Hcy are common in the general population, particularly in the elderly. Vitamin status is a

\section{References}

1. Selhub J. Homocysteine metabolism. Annu Rev Nutr 1999; 19: 217-246.

2. Mansoor MA, Bergmark C, Svardal AM, Lonning PE, Ueland PM. Redox status and protein binding of plasma homocysteine and other aminothiols in patients with early-onset peripheral vascular disease. Homocysteine and peripheral primary determinant of mild-to-moderate HHcy and accounts for approximately two thirds of all such cases. Vitamin supplementation results in near normalization of plasma Hcy in most cases. Hcy metabolism requires the participation of folate as well as vitamin B12 (cobalamin) and vitamin B6 (pyridoxal phosphate) coenzymes and, therefore, reduction of Hcy levels in plasma requires supplementation with all three vitamins (1). The minimum daily requirement of folic acid is $50 \mu \mathrm{g}$, although the current recommended intake is $400 \mu \mathrm{g} /$ day for the average adult and $600 \mu \mathrm{g} /$ day during pregnancy (56).

A large study on 750 males observed that dietary intervention with increased focus and availability of vegetables, fruits and bread, significantly reduced the concentration of tHcy. These findings suggest that the changes in the concentration of cysteine, folate and flavin mononucleotide seem to be predictors of changes in tHcy concentration (57).

Fortification of grain food with folic acid to decrease the incidence of NTD was started in the USA and Canada in 1998. This public health strategy reduced tHcy concentrations, leading not only to a decrease of the prevalence of congenital anomalies but also to decline in the incidence of stroke mortality (58).

In the last decade several studies have been conducted to uncover the direct or indirect influence of increased Hcy levels in several conditions.

It was demonstrated that several polymorphisms in genes of the Hcy-methionine pathway result in HHcy, suggesting that these variants may play a role in many multifactorial disorders of high prevalence in the general population.

Vitamin supplementation is a cost-effective way to decrease HHcy and would prevent at least some of these disorders.

Despite these robust indications about the role of HHcy as a factor associated with several conditions, long-term studies on large samples are still lacking to prove that decreasing the Hcy levels in subjects with HHcy results in better results in terms of health outcomes.

\section{Acknowledgments}

Research supported by FIRCA/NIH TW007644-02 (T.M. Félix) and CNPq. R. Giugliani and S. Brustolin are recipients of fellowships from CNPq. vascular disease. Arterioscler Thromb Vasc Biol 1995; 15: 232-240.

3. Frosst P, Blom HJ, Milos R, Goyette P, Sheppard CA, Matthews $R G$, et al. A candidate genetic risk factor for vascular disease: a common mutation in methylenetetrahydrofolate reductase. Nat Genet 1995; 10: 111-113. 
4. Jacques PF, Bostom AG, Williams RR, Ellison RC, Eckfeldt $\mathrm{JH}$, Rosenberg IH, et al. Relation between folate status, a common mutation in methylenetetrahydrofolate reductase, and plasma homocysteine concentrations. Circulation 1996; 93: 7-9.

5. van der Put NM, Gabreels F, Stevens EM, Smeitink JA, Trijbels FJ, Eskes TK, et al. A second common mutation in the methylenetetrahydrofolate reductase gene: an additional risk factor for neural-tube defects? Am J Hum Genet 1998; 62: 1044-1051.

6. Rozen R. Genetic predisposition to hyperhomocysteinemia: deficiency of methylenetetrahydrofolate reductase (MTHFR). Thromb Haemost 1997; 78: 523-526.

7. Metz J, Bell AH, Flicker L, Bottiglieri T, Ibrahim J, Seal E, et al. The significance of subnormal serum vitamin B12 concentration in older people: a case control study. J Am Geriatr Soc 1996; 44: 1355-1361.

8. Nurk E, Tell GS, Nygard O, Refsum H, Ueland PM, Vollset SE. Plasma total homocysteine is influenced by prandial status in humans: the Hordaland Homocysteine Study. $J$ Nutr 2001; 131: 1214-1216.

9. Weiss N, Keller C, Hoffmann U, Loscalzo J. Endothelial dysfunction and atherothrombosis in mild hyperhomocysteinemia. Vasc Med 2002; 7: 227-239.

10. Berry RJ, Li Z, Erickson JD, Li S, Moore CA, Wang H, et al. Prevention of neural-tube defects with folic acid in China. China-U.S. Collaborative Project for Neural Tube Defect Prevention. N Engl J Med 1999; 341: 1485-1490.

11. Steegers-Theunissen RP, Boers GH, Trijbels FJ, Finkelstein JD, Blom HJ, Thomas CM, et al. Maternal hyperhomocysteinemia: a risk factor for neural-tube defects? Metabolism 1994; 43: 1475-1480.

12. Mills JL, McPartlin JM, Kirke PN, Lee YJ, Conley MR, Weir DG, et al. Homocysteine metabolism in pregnancies complicated by neural-tube defects. Lancet 1995; 345: 149-151.

13. van der Put NM, van der Molen EF, Kluijtmans LA, Heil SG, Trijbels JM, Eskes TK, et al. Sequence analysis of the coding region of human methionine synthase: relevance to hyperhomocysteinaemia in neural-tube defects and vascular disease. QJM 1997; 90: 511-517.

14. Wenstrom KD, Johanning GL, Owen J, Johnston KE, Acton $S$, Cliver $S$, et al. Amniotic fluid homocysteine levels, 5,10-methylenetetrahydrofolate reductase genotypes, and neural tube closure sites. Am J Med Genet 2000; 90: 6-11.

15. Ueland PM, Hustad S, Schneede J, Refsum H, Vollset SE. Biological and clinical implications of the MTHFR C677T polymorphism. Trends Pharmacol Sci 2001; 22: 195-201.

16. Epeldegui M, Pena-Melian A, Varela-Moreiras G, PerezMiguelsanz J. Homocysteine modifies development of neurulation and dorsal root ganglia in chick embryos. Teratology 2002; 65: 171-179.

17. Ratan SK, Rattan KN, Pandey RM, Singhal S, Kharab S, Bala M, et al. Evaluation of the levels of folate, vitamin B12, homocysteine and fluoride in the parents and the affected neonates with neural tube defect and their matched controls. Pediatr Surg Int 2008; 24: 803-808.

18. Felix TM, Leistner S, Giugliani R. Metabolic effects and the methylenetetrahydrofolate reductase (MTHFR) polymorphism associated with neural tube defects in southern Brazil. Birth Defects Res A Clin Mol Teratol 2004; 70: 459-463.

19. Perez AB, D'Almeida V, Vergani N, de Oliveira AC, de Lima FT, Brunoni D. Methylenetetrahydrofolate reductase (MTH-
FR): incidence of mutations C677T and A1298C in Brazilian population and its correlation with plasma homocysteine levels in spina bifida. Am J Med Genet A 2003; 119A: 2025.

20. Wong WY, Eskes TK, Kuijpers-Jagtman AM, Spauwen PH, Steegers EA, Thomas CM, et al. Nonsyndromic orofacial clefts: association with maternal hyperhomocysteinemia. Teratology 1999; 60: 253-257.

21. Wilcox AJ, Lie RT, Solvoll K, Taylor J, McConnaughey DR, Abyholm $\mathrm{F}$, et al. Folic acid supplements and risk of facial clefts: national population based case-control study. BMJ 2007; 334: 464 .

22. Shotelersuk V, Ittiwut $C$, Siriwan $P$, Angspatt A. Maternal 677CT/1298AC genotype of the MTHFR gene as a risk factor for cleft lip. J Med Genet 2003; 40: e64.

23. Verkleij-Hagoort A, Bliek J, Sayed-Tabatabaei F, Ursem N, Steegers E, Steegers-Theunissen R. Hyperhomocysteinemia and MTHFR polymorphisms in association with orofacial clefts and congenital heart defects: a meta-analysis. $A m \mathrm{~J}$ Med Genet A 2007; 143A: 952-960.

24. Botto LD, Correa A, Erickson JD. Racial and temporal variations in the prevalence of heart defects. Pediatrics 2001; 107: E32.

25. Trabetti E. Homocysteine, MTHFR gene polymorphisms, and cardio-cerebrovascular risk. J Appl Genet 2008; 49: 267-282.

26. Burke AP, Fonseca V, Kolodgie F, Zieske A, Fink L, Virmani $R$. Increased serum homocysteine and sudden death resulting from coronary atherosclerosis with fibrous plaques. Arterioscler Thromb Vasc Biol 2002; 22: 1936-1941.

27. Zhou J, Moller J, Ritskes-Hoitinga M, Larsen ML, Austin $R C$, Falk E. Effects of vitamin supplementation and hyperhomocysteinemia on atherosclerosis in apoE-deficient mice. Atherosclerosis 2003; 168: 255-262.

28. Wang $H$, Jiang $X$, Yang F, Gaubatz JW, Ma L, Magera MJ, et al. Hyperhomocysteinemia accelerates atherosclerosis in cystathionine beta-synthase and apolipoprotein $\mathrm{E}$ double knock-out mice with and without dietary perturbation. Blood 2003; 101: 3901-3907.

29. Matthias D, Becker $\mathrm{CH}$, Riezler R, Kindling PH. Homocysteine induced arteriosclerosis-like alterations of the aorta in normotensive and hypertensive rats following application of high doses of methionine. Atherosclerosis 1996; 122: 201-216.

30. Zhou J, Werstuck GH, Lhotak S, Shi YY, Tedesco V, Trigatti $B$, et al. Hyperhomocysteinemia induced by methionine supplementation does not independently cause atherosclerosis in C57BL/6J mice. FASEB J 2008; 22: 2569-2578.

31. Bosco P, Gueant-Rodriguez RM, Anello G, Barone C, Namour F, Caraci F, et al. Methionine synthase (MTR) 2756 $(A \longrightarrow G)$ polymorphism, double heterozygosity methionine synthase $2756 \mathrm{AG} /$ methionine synthase reductase (MTRR) $66 \mathrm{AG}$, and elevated homocysteinemia are three risk factors for having a child with Down syndrome. Am J Med Genet A 2003; 121A: 219-224.

32. Chango A, Fillon-Emery N, Mircher $\mathrm{C}$, Blehaut $\mathrm{H}$, Lambert $D$, Herbeth $B$, et al. No association between common polymorphisms in genes of folate and homocysteine metabolism and the risk of Down's syndrome among French mothers. $\mathrm{Br}$ J Nutr 2005; 94: 166-169.

33. da Silva LR, Vergani N, Galdieri LC, Ribeiro Porto MP, Longhitano SB, Brunoni D, et al. Relationship between poly- 
morphisms in genes involved in homocysteine metabolism and maternal risk for Down syndrome in Brazil. Am J Med Genet A 2005; 135: 263-267.

34. Biselli JM, Goloni-Bertollo EM, Zampieri BL, Haddad R, Eberlin MN, Pavarino-Bertelli EC. Genetic polymorphisms involved in folate metabolism and elevated plasma concentrations of homocysteine: maternal risk factors for Down syndrome in Brazil. Genet Mol Res 2008; 7: 33-42.

35. Marszall M, Czarnowski W. [Smoking influence on the level of homocysteine and 5-methyltetrahydrofolic acid in active and non smokers]. Przegl Lek 2007; 64: 685-688.

36. Sobczak A, Szoltysek-Boldys I, Grela W, Zielinska-Danch W. [The influence of tobacco smoke on homocysteine level in plasma of healthy males]. Przegl Lek 2007; 64: 679-684.

37. Walker MC, Smith GN, Perkins SL, Keely EJ, Garner PR. Changes in homocysteine levels during normal pregnancy. Am J Obstet Gynecol 1999; 180: 660-664.

38. Ambroszkiewicz J, Chelchowska M, Lewandowski L, Gajewska J, Laskowska-Klita T. [Serum folate and homocysteine concentrations in women smoking during pregnancy and in umbilical cord blood of newborns]. Przegl Lek 2007; 64: 674-678.

39. Ravaglia G, Forti P, Maioli F, Martelli M, Servadei L, Brunetti $\mathrm{N}$, et al. Homocysteine and folate as risk factors for dementia and Alzheimer disease. Am J Clin Nutr 2005; 82: 636-643.

40. Koseoglu E, Karaman Y. Relations between homocysteine, folate and vitamin B12 in vascular dementia and in Alzheimer disease. Clin Biochem 2007; 40: 859-863.

41. Durga J, van Boxtel MP, Schouten EG, Kok FJ, Jolles J, Katan MB, et al. Effect of 3-year folic acid supplementation on cognitive function in older adults in the FACIT trial: a randomised, double blind, controlled trial. Lancet 2007; 369: 208-216.

42. Duthie SJ. Folic acid deficiency and cancer: mechanisms of DNA instability. Br Med Bull 1999; 55: 578-592.

43. Lewis SJ, Harbord RM, Harris R, Smith GD. Meta-analyses of observational and genetic association studies of folate intakes or levels and breast cancer risk. J Natl Cancer Inst 2006; 98: 1607-1622.

44. Lissowska J, Gaudet MM, Brinton LA, Chanock SJ, Peplonska B, Welch R, et al. Genetic polymorphisms in the one-carbon metabolism pathway and breast cancer risk: a population-based case-control study and meta-analyses. Int J Cancer 2007; 120: 2696-2703.

45. Suzuki T, Matsuo K, Hirose K, Hiraki A, Kawase T, Watanabe $M$, et al. One-carbon metabolism-related gene polymorphisms and risk of breast cancer. Carcinogenesis 2008; 29: 356-362.

46. Bjelland I, Tell GS, Vollset SE, Refsum H, Ueland PM. Folate, vitamin B12, homocysteine, and the MTHFR $677 \mathrm{C} \longrightarrow$ T polymorphism in anxiety and depression: the Hordaland
Homocysteine Study. Arch Gen Psychiatry 2003; 60: 618626.

47. Almeida OP, Flicker L, Lautenschlager NT, Leedman P, Vasikaran S, van Bockxmeer FM. Contribution of the MTHFR gene to the causal pathway for depression, anxiety and cognitive impairment in later life. Neurobiol Aging 2005; 26 : 251-257.

48. Tiemeier H, van Tuijl HR, Hofman A, Meijer J, Kiliaan AJ, Breteler MM. Vitamin B12, folate, and homocysteine in depression: the Rotterdam Study. Am J Psychiatry 2002; 159: 2099-2101.

49. Kim JM, Stewart R, Kim SW, Yang SJ, Shin IS, Yoon JS. Predictive value of folate, vitamin B12 and homocysteine levels in late-life depression. Br J Psychiatry 2008; 192: 268-274.

50. Vaccaro O, Perna AF, Mancini FP, lovine C, Cuomo V, Sacco $\mathrm{M}$, et al. Plasma homocysteine and microvascular complications in type 1 diabetes. Nutr Metab Cardiovasc Dis 2000; 10: 297-304.

51. Martinez Laborda S, Salazar Garcia-Blanco MI, Rodriguez Rigual M, Baldellou Vazquez A. [Total homocysteine levels in children with diabetes type 1. Conditional factors]. An Pediatr 2008; 68: 264-268.

52. Wotherspoon F, Laight DW, Turner C, Meeking DR, Allard SE, Munday LJ, et al. The effect of oral folic acid upon plasma homocysteine, endothelial function and oxidative stress in patients with type 1 diabetes and microalbuminuria. Int J Clin Pract 2008; 62: 569-574.

53. Apeland T, Mansoor MA, Pentieva K, McNulty H, Seljeflot I, Strandjord RE. The effect of B-vitamins on hyperhomocysteinemia in patients on antiepileptic drugs. Epilepsy Res 2002; 51: 237-247.

54. Sener U, Zorlu Y, Karaguzel O, Ozdamar O, Coker I, Topbas M. Effects of common anti-epileptic drug monotherapy on serum levels of homocysteine, vitamin B12, folic acid and vitamin B6. Seizure 2006; 15: 79-85.

55. Belcastro V, Gaetano G, Italiano D, Oteri G, Caccamo D, Pisani LR, et al. Antiepileptic drugs and MTHFR polymorphisms influence hyper-homocysteinemia recurrence in epileptic patients. Epilepsia 2007; 48: 1990-1994.

56. McCully KS. Homocysteine, vitamins, and vascular disease prevention. Am J Clin Nutr 2007; 86: 1563S-1568S.

57. Stea TH, Mansoor MA, Wandel M, Uglem S, Frolich W. Changes in predictors and status of homocysteine in young male adults after a dietary intervention with vegetables, fruits and bread. Eur J Nutr 2008; 47: 201-209.

58. Yang Q, Botto LD, Erickson JD, Berry RJ, Sambell C, Johansen $\mathrm{H}$, et al. Improvement in stroke mortality in Canada and the United States, 1990 to 2002. Circulation 2006; 113: 1335-1343. 